\title{
Evolutionary sequences of stellar models with new radiative opacities. \\ VI. $Z=0.0001$
}

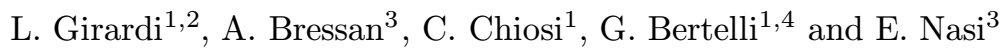 \\ 1 Department of Astronomy, Vicolo Osservatorio 5, 35122 Padua, Italy \\ 2 Instituto de Física, UFRGS, C.P. 15051, 91501-970 Porto Alegre RS, Brazil \\ 3 Astronomical Observatory, Vicolo Osservatorio 5, 35122 Padua, Italy \\ ${ }^{4}$ Fellow of the National Council of Research CNR
}

Received July 28; accepted November 7, 1995

\begin{abstract}
We present a large grid of stellar evolutionary models with the initial chemical composition $[Z=$ $0.0001, Y=0.23]$. These tracks are conceived to extend the grid of stellar models described in the previous papers of this series, and are computed with the new radiative opacities by Iglesias et al. (1992) and convective overshoot. The tracks span the range of initial masses from $0.6 M_{\odot}$ to $100 M_{\odot}$, and extend from the zero age main sequence (ZAMS) till very advanced evolutionary phases. Specifically, low- and intermediate-mass stars are followed till the beginning of the thermally pulsing regime of the asymptotic red giant branch phase (TP-AGB), while massive stars are followed till the core C-ignition. With respect to previous papers of this series, these models incorporate a number of small modifications in the input physics, particularly on the equation of state, which now incorporates the effect of Coulomb interactions between charged particles. The effect of these modifications is discussed. The corresponding theoretical isochrones are presented.
\end{abstract}

Key words: stars: evolution — stars: interiors — stars: Hertzsprung-Russel (HR) diagram — stars: abundances

\section{Introduction}

This is a sequel of the series of papers aimed at providing a database of stellar models which has to be firstly complete in mass, chemical composition, and major evolutionary stages, and secondly homogeneous as well as updated in its physical ingredients.

Let us briefly recall the main characteristics of the stellar tracks so far presented in this series. It started with the study by Alongi et al. (1993) presenting grids with chemical composition $[Y=0.250, Z=0.008]$, adopting both the classical and the overshoot schemes for the treatment of convection in central cores and external envelopes. The opacity in use was the classical radiative opacity by Huebner et al. (1977, thereinafter LAOL). Afterwards, LAOL opacities were superseded by the results of the Livermore group (Rogers \& Iglesias 1992; Iglesias et al. 1992, thereinafter OPAL), and tracks incorporating the new OPAL opacities were presented for the chemical compositions $[Y=0.280, Z=0.020]$ (Bressan et al. 1993a), $[Y=0.230, Z=0.0004]$ and $[Y=0.352, Z=0.05]$

Send offprint requests to: C. Chiosi
(Fagotto et al. 1994a), and $[Y=0.240, Z=0.004]$ and $[Y=0.250, Z=0.008]$ (Fagotto et al. 1994b). An additional grid of stellar tracks with $[Y=0.475, Z=0.1]$ and use of the LAOL opacities was calculated by Fagotto et al. (1994c).

From all the above models, the grids presented by Bressan et al. (1993a) and Fagotto et al. (1994a, b) perfectly satisfy the request of homogeneity, physical accuracy and coverage of evolutionary stages that was outlined in the first study by Alongi et al. (1993) and stressed in the subsequent papers. They gave origin to the large set of isochrones calculated by Bertelli et al. (1994), and so far were used for a series of purposes, from the study of CMDs of star clusters and nearby galaxies to the study of the spectrophotometric properties of bulges and elliptical galaxies (e.g. Bressan et al. 1994b; Girardi et al. 1995a).

In this paper, we present a grid of stellar models with initial chemical composition $[Y=0.230, Z=0.0001]$. They are primarily conceived to extend, into the lowermetallicity end, the set of stellar tracks available at Padua.

Due to a number of improvements on the input physics, these models do not perfectly meet the requirements of homogeneity with the previous grids calculated by Bressan 
et al. (1993a) and Fagotto et al. (1994a, b). However, it will be shown that these changes in the input physics are not able to modify in a systematic or dramatic way the path described by the models on the HRD. Therefore, the set of isochrones that we calculate and present in this paper can be safely used together with those by Bertelli et al. (1994).

The description of the models follows. We put emphasis in the description of the input physics which was improved with respect to the previous papers of this series, and on the particularities of the evolution which arise at this extremely low value for the initial metallicity.

As usual the evolutionary tracks are presented sampled in three groups, in which the stars share similar properties. Specifically, low-mass stars $\left(M \leq M_{\mathrm{HeF}}\right)$ are those which develop a degenerate helium core and ignite helium through a phase of violent burning episodes (HeliumFlash), intermediate-mass stars are those which avoid core He-flash but develop a highly electron-degenerate carbonoxygen core $\left(M_{\mathrm{HeF}} \leq M \leq M_{\mathrm{up}}\right)$ in which carbon ignites violently, and massive stars $\left(M \geq M_{\text {up }}\right)$ as those which are able to avoid degeneracy till core C-ignition.

\section{Physical ingredients of the models}

As in the previous papers, the helium content at the limit of low metallicities is assumed to be $Y=0.23$.

As far as the opacity, the mixing scheme, and the massloss rates are concerned, the physical input is the same as that used by Bressan et al. (1993a) and Fagotto et al. (1994a, b) according to the request of homogeneity as previously claimed. Therefore, we will limit ourselves to briefly recall only the assumptions concerning these ingredients.

The radiative opacity is from the Livermore Library (Iglesias et al. 1992) which supersedes the previous OPAL computations (Rogers \& Iglesias 1992 and references) because of the inclusion of the spin-orbit interaction in the treatment of $\mathrm{Fe}$ atomic data and the adoption of recent measurements of the solar photospheric abundance of Fe. To be more precise, we use the latest (1993) release of OPAL, which differs from the Iglesias et al. (1992) release of only $1-2 \%$ percent, due to the adoption of Grevesse \& Noels (1993) solar composition instead of Grevesse (1991). For the outermost regions the radiative opacities are implemented with those of the LAOL and the molecular contribution according to the prescription by Bessell et al. $(1989,1991)$. We notice that at such low metallicities the contribution from metallic molecules to the opacities is very small.

The full network of nuclear reactions which is followed is the same as in Bressan et al. (1993a). Reaction rates are from Caughlan \& Fowler (1988), but for: (1) the reactions ${ }^{17} \mathrm{O}(\mathrm{p}, \alpha){ }^{14} \mathrm{~N}$ and ${ }^{17} \mathrm{O}(\mathrm{p}, \gamma){ }^{18} \mathrm{~F}$ in which we use the more recent determinations by Landré et al. (1990); and (2) ${ }^{12} \mathrm{C}(\alpha, \gamma){ }^{16} \mathrm{O}$, which rate was set to be 1.7 times that given by Caughlan \& Fowler (1988), as indicated by the study by Weaver \& Woosley (1993) on the nucleosynthesis by massive stars.

The equation of state (EOS) has been revised. For the interior, the EOS is that of a fully-ionized gas, including electron degeneracy in the way described by Kippenhahn et al. (1965). We included the effect of Coulomb interactions between the gas particles at high densities following mainly the formulation by Straniero (1988). We however adapted this formulation to the general case of a multiplecomponent plasma, thus allowing us to explicitly calculate the Coulomb contribution to the thermodynamical variables at every mesh point of the models. Details are given in the Appendix.

For the stellar envelope, thermodynamical quantities are derived from a H-He mixture with ionization equilibrium determined by a simple set of Saha equations (Baker \& Kippenhahn 1962). Comparison of the thermodynamical quantities with those derived from the free-energy minimization technique of Mihalas et al. (1990 and references therein) reveals that it is a good approximation as long as the formation of the $\mathrm{H}_{2}$ molecule is not important. This condition is perfectly met in most of the stellar tracks presented in this paper; the only one which could be affected by $\mathrm{H}_{2}$ formation is the $0.6 M_{\odot}$ at the MS stage. We are presently working on the extension of the present stellar models to very-low masses (Girardi et al. 1995b), and anticipate that the effect of the $\mathrm{H}_{2}$ molecule on the MS position of a $0.6 M_{\odot}$ star is already negligible.

As already mentioned the models are calculated evaluating the extension of the convective regions (wherever necessary) by means of the overshoot scheme of mixing according to the prescriptions given by Bressan et al. (1981), Bertelli et al. (1985), Alongi et al. (1991, 1993), Bressan et al. (1993a), and Fagotto et al. (1993a). The efficiency of this process has been preliminarily calibrated on observational data for LMC clusters and Galactic old open clusters. Current estimates indicate a mild efficiency of core overshoot, or $\Lambda_{\mathrm{c}} \leq 0.5$, and of envelope overshoot, or $\Lambda_{\mathrm{e}}=0.7$ (see the discussion of this topic by Chiosi et al. 1992a).

Although significant mass loss by stellar wind may occur during the red giant (RGB) and asymptotic giant branch (AGB) phases of low- and intermediate-mass stars, the models are calculated at constant mass. This is possible because of the regular behaviour of the RGB and AGB phases for the adopted chemical compositions, so that mass loss by stellar wind can be included by means of the analytical method outlined long ago by Renzini (1977) and since then adopted in many studies (e.g. Bertelli et al. 1990, 1994, see also Sect. 5) when isochrones are constructed from evolutionary sequences. In brief, going from the tip of the RGB to the zero age horizontal branch (ZAHB) the mass of the star is simply scaled by removing a fraction of the envelope. Past the onset of the TP-AGB 
phase, the evolution can be followed analytically provided that the relationship between the mass of the $\mathrm{H}$-exhausted core and the total luminosity, and the rate of mass loss as a function of basic stellar parameters (e.g. luminosity, mass, and radius) are assigned.

On the contrary, massive stars with initial mass $M \geq$ $12 M_{\odot}$ are always evolved including the effect of mass loss by stellar wind from the ZAMS stage because their structure and evolution is entirely dominated by this phenomenon (Chiosi \& Maeder 1986; Chiosi et al. 1992a, b). The empirical mass-loss rates are the same as in Bressan et al. (1994a) and Fagotto et al. (1994a). These are based upon observational data for supergiants, luminous blue variables (LBV), and Wolf Rayet (WR) stars. Precisely, for all evolutionary stages from the main sequence up to the so-called de Jager limit in the Hertzsprung Russell Diagram (HRD), the mass-loss rates are derived from de Jager et al. (1988) however modulated on the metallicity according to the prescription by Kudritzki et al. (1989). The mass-loss rates are expressed as

$$
\dot{M}_{Z}=Z^{0.5} \times \dot{M}_{Z=0.02}
$$

Beyond the de Jager limit, the mass-loss rate $\dot{M}_{Z=0.02}$ is increased to $10^{-3} M_{\odot} \mathrm{yr}^{-1}$ as suggested by the observational data for the LBV stars.

\section{The mixing length calibration}

Since some physical ingredients of the models have been changed, we are forced to revise the calibration of the mixing length parameter $\alpha=l / H_{\mathrm{p}}$ in the outermost super-adiabatic convective region. With the introduction of Coulomb interactions in the EOS, models turned to be denser and brighter than their counterparts which did not consider these terms (see the Appendix). In consequence, the model which previously fitted the luminosity and radius of the Sun at the solar age, now should have its $\alpha$ increased and He content decreased in order to satisfy the same constraints. We find that a $1.0 M_{\odot}$ model with $Z=0.01886$ (the solar $Z$ according to Anders \& Grevesse $1989)$ reproduces the solar radius and luminosity at an age of 4.6 Gyr only if we assume $\alpha=1.56$ and $Y=0.273$. This value for $\alpha$ replaces that used in the previous papers of this series.

It is worth emphasizing that the use of a different value of $\alpha$ does not constitute a serious source of heterogeneity among the different sets of tracks presented in this series of papers. Apart from the fact that $\alpha$ can only affect models within a limited range of effective temperatures, the modifications in the stellar structure were small enough such that the small changes in effective temperature and luminosity of the models were almost completely compensated by the changes made in order to recalibrate the solar model. Therefore, the new models turn to follow almost the same track on the HRD as before. It is illustrated in Fig. 1, in which we superimpose two different sets of low-mass stellar models: those by Bressan et al. (1993a), and a limited set of tracks computed with initial composition $[Z=0.01886, Y=0.273]$ and the same input physics as described in this section. It is evident that both sets almost perfectly superimpose in the HRD, all along from the main sequence to the red giant phases. Temperature differences between any two equivalent tracks are always lower than $60 \mathrm{~K}$. A similar test was performed on a limited set of tracks with $[Y=0.23, Z=0.0004]$ and $[Y=0.475, Z=0.05]$, compared to the tracks presented by Fagotto et al. (1994a), with identical results.

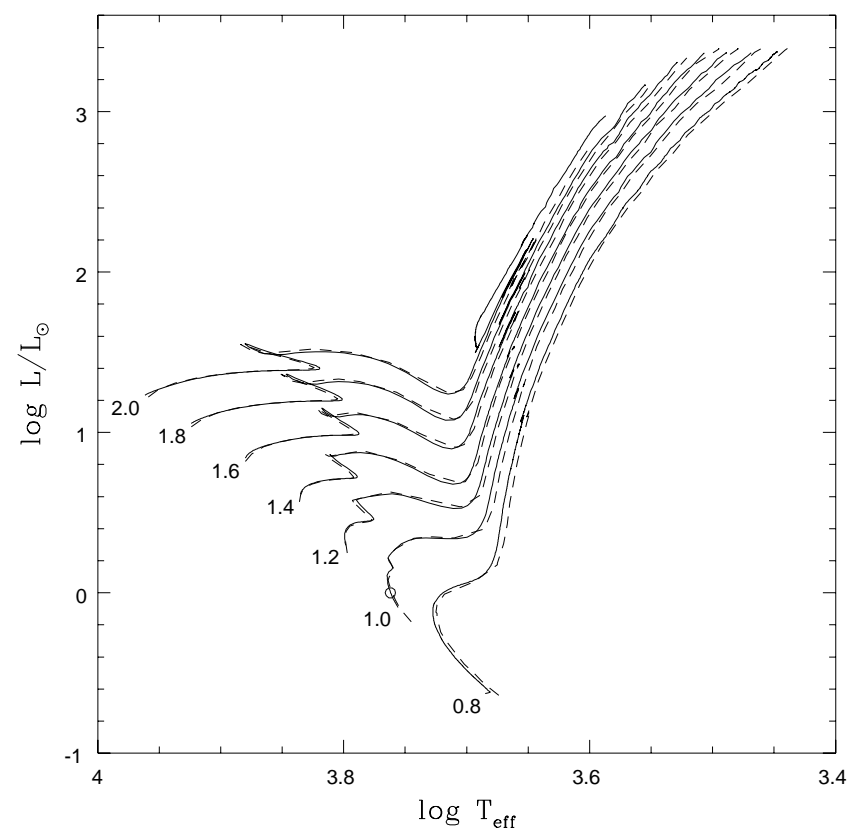

Fig. 1. Theoretical HRD presenting two different sets of tracks for low-mass stars: a sample of those calculated by Bressan et al. (1993a) with $[Z=0.02, Y=0.28]$ (dashed lines), and another one computed with $[Z=0.01886, Y=0.273]$ and a slightly different input physics (this paper; solid lines). Both sets are calibrated to the Sun (circle). The mass of each star in solar units is indicated. The tracks go from the ZAMS to the tip of the RGB, except for the $2 M_{\odot}$ star with new input physics, which starts burning $\mathrm{He}$ at the center in non-degenerate conditions and goes till the beginning of the TP-AGB phase

The main effect of the introduction of Coulomb interactions in the EOS is on the lifetimes of low-mass stars. Figure 2 illustrates the change in lifetimes from the ZAMS to the beginning of core He-burning, for tracks of two different chemical compositions, with respect to the tracks presented by Bressan et al. (1993a) and Fagotto et al. (1994a). As expected, the change in lifetime is almost null for a $1 M_{\odot}$ star with solar composition, and differences of a few percent arise for the remaining stars. For the models with near-solar composition, lifetimes differ by at most $5 \%$, an effect that could be safely neglected in the study of young and intermediate-age stellar populations. For $Z=0.0004$, however, a more dramatic change occurs 


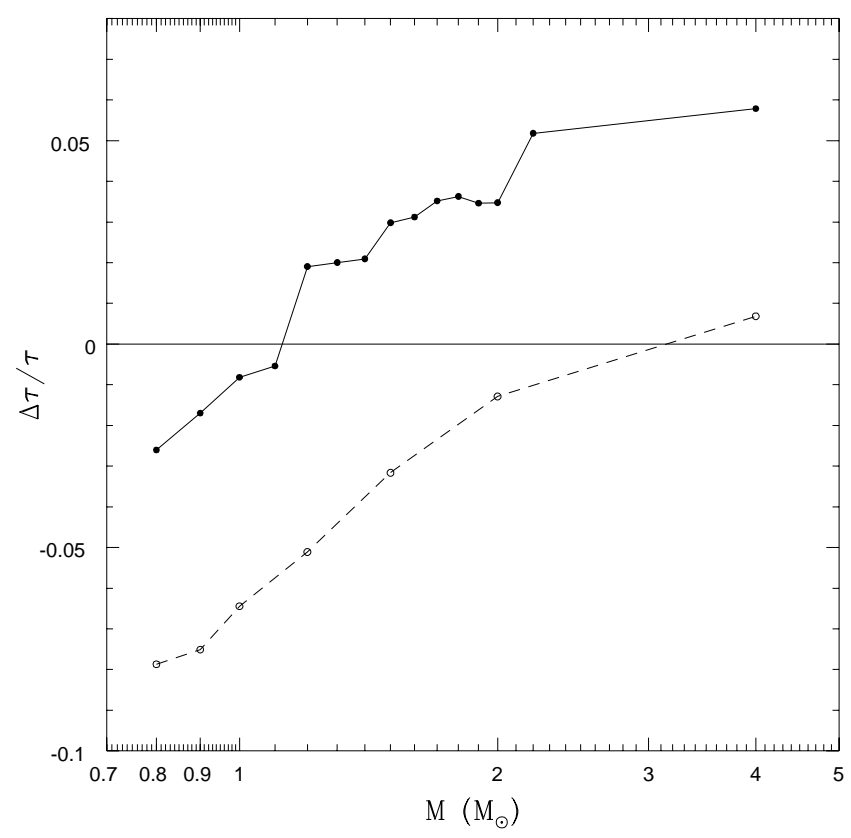

Fig. 2. The fractional increase in lifetimes from the ZAMS to the start of central He-burning, due to the changes on the input physics of our models, as a function of stellar mass. Solid line and full dots: difference between a set of tracks computed with $[Z=0.01886, Y=0.273]$ and the input physics described in this paper, and the tracks of Bressan et al. (1993a; see Fig. 1). Dashed line and dots: difference between tracks computed with $[Z=0.0004, Y=0.23]$ and the input physics described in this paper, and Fagotto et al. (1994a) tracks for the same chemical composition

at the low-mass end: a $0.8 M_{\odot}$ star decreases its lifetime of $8 \%$, without any appreciable effect in the turn-off luminosity. It is a small but not negligible effect, since it would imply a decrease of $\sim 1 \mathrm{Gyr}$ in the absolute age estimates of globular clusters made with our isochrones.

\section{Evolutionary results}

In the following we make a short discussion on some global features of the HRDs, lifetimes, and lifetime ratios of the major evolutionary phases, and the variation of the surface abundance induced by dredge-up and mass loss.

We inform the reader that complete tables containing all the relevant information on the models (the same described in previous papers of this series), can be obtained upon request to the authors. The data is also available in a public directory at Padua, and is accessible through the World Wide Web (WWW) at the node http://www.pd.astro.it.

Figures 3, 4 and 5 show the corresponding HRDs for the low mass range (stars undergoing core He-flash), from the ZAMS to the tip of the RGB, and from the ZAHB to the start of the TP-AGB phase. For the sake of clarity, the set of tracks from the ZAHB to later phases is presented in two different figures: Fig. 4 with ZAHB mass in the range $0.5-0.8 M_{\odot}$, and Fig. 5 displaying the same but for ZAHB masses in the range $0.9-1.7 M_{\odot}$.

Looking at Fig. 3, the reader can notice the main features of the low-metallicity grid of low-mass evolutionary tracks. Particularly interesting are the narrowness of the main sequence band, the large temperature range covered by the SGBs, and the almost vertical track of the RGB. The transition mass from low to intermediate mass stars is $M_{\mathrm{HeF}}=1.7 M_{\odot}$.

During the core He-burning and later phases of low mass stars, the evolutionary path in the HRD develops at the high temperatures characteristic of low metallicity models. The ZAHB is shifted to higher and higher temperatures at both decreasing and increasing ZAHB mass (Figs. 4 and 5, respectively). Following core He-exhaustion the models evolve toward the Hayashi line to start the AGB phase. The $0.51 M_{\odot}$ model, however, settles on very hot temperatures and proceeds exhausting the central fuel growing its luminosity and maintaining its high temperature. A pronounced He-shell flash takes place when the star has not yet settled on the Hayashi line.

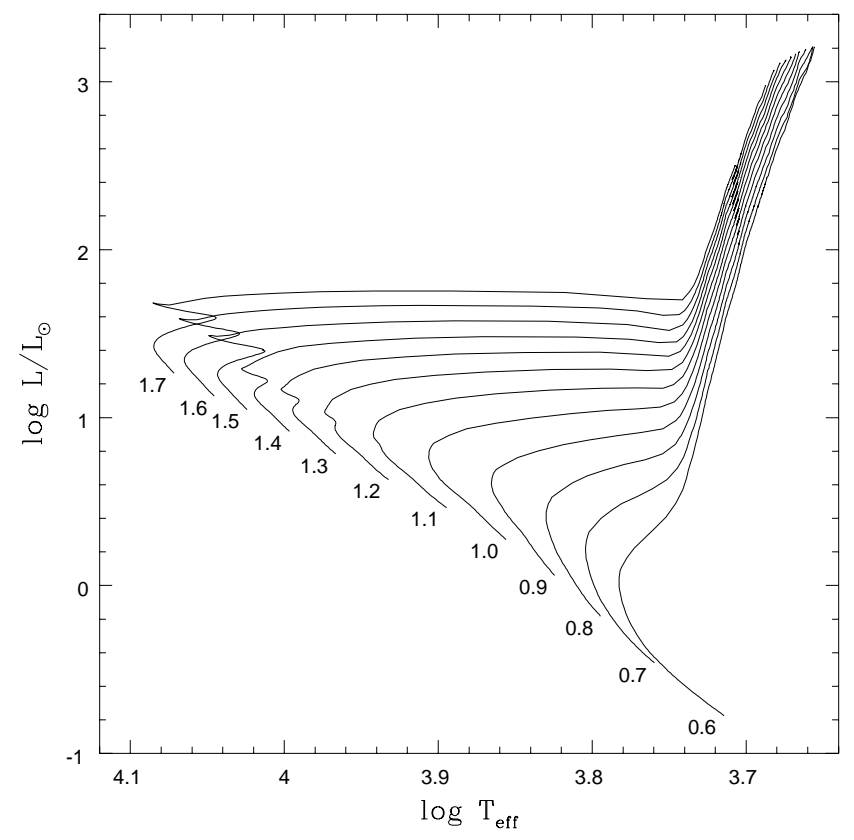

Fig. 3. Theoretical HRD for the low mass stars with composition $Y=0.230$ and $Z=0.0001$. Each evolutionary track goes from the ZAMS to the tip of the RGB. Models, in which during the core H-burning phase a convective core can develop, are calculated with mild overshoot. The parameter $\Lambda_{\mathrm{c}}$ is 0.25 in the mass range $1.0 M_{\odot} \leq M \leq 1.4 M_{\odot}$ and 0.5 above it. Overshoot at the bottom of the convective envelope along the Hayashi line is calculated with $\Lambda_{\mathrm{e}}=0.7$. The initial mass in solar unit is indicated along each curve

Figure 6 presents the HRD for intermediate mass stars and those massive stars of lower mass in which the effect of 


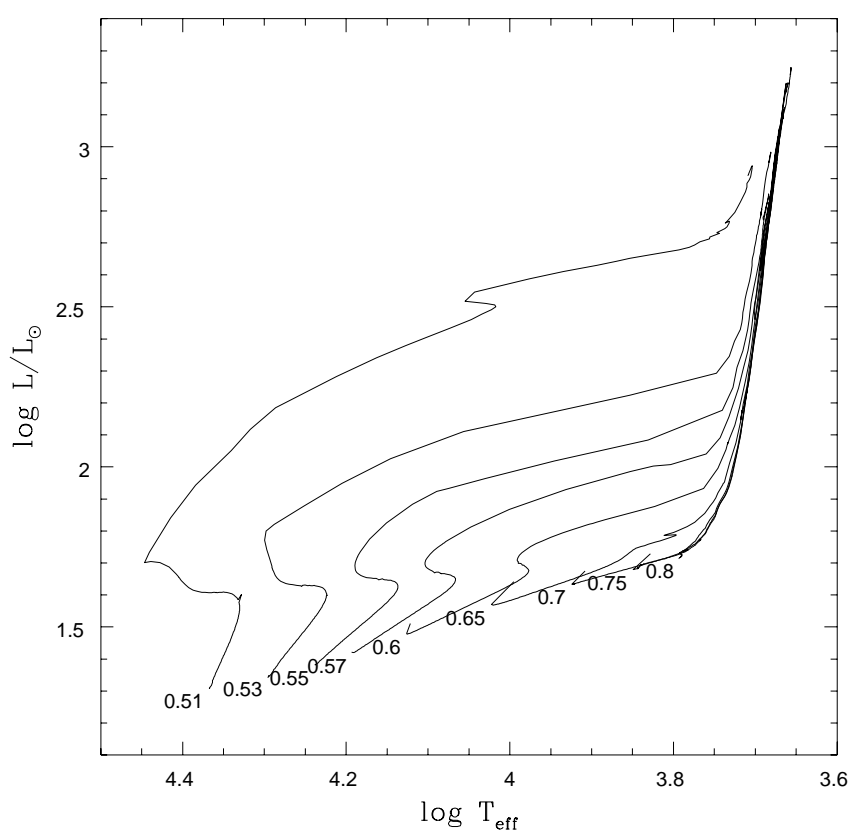

Fig. 4. Theoretical HRD for low-mass stars with ZAHB mass between 0.5 and $0.8 M_{\odot}$, during the core He-burning phase. The models go from the ZAHB to the beginning of the TP-AGB phase, and are calculated with the overshoot scheme. The mass of the stars in solar units is indicated along each evolutionary track

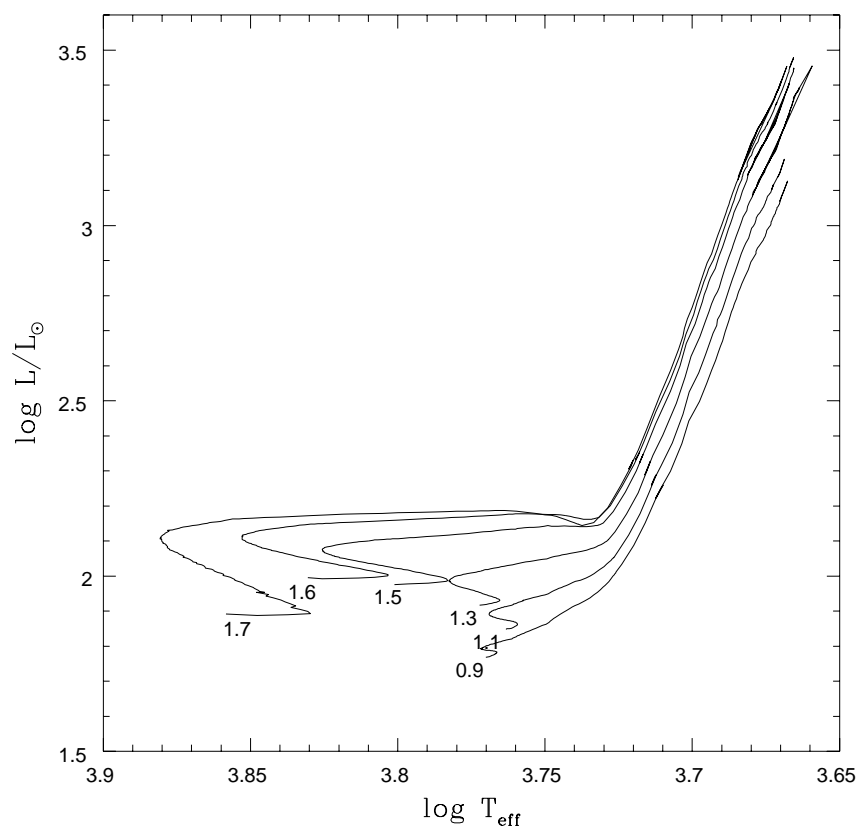

Fig. 5. Same as in Fig. 4, but for low-mass stars with ZAHB mass between 0.9 and $1.7 M_{\odot}$. For the sake of clarity, the tracks for ZAHB masses of $1.0,1.2$, and $1.4 M_{\odot}$, also available, are not presented

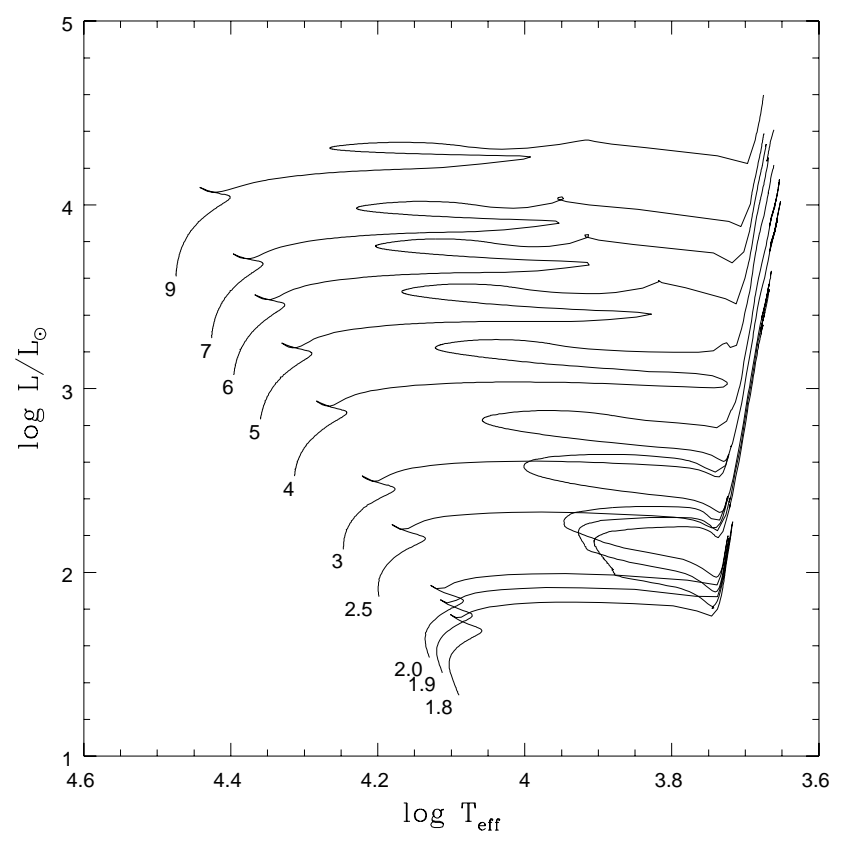

Fig. 6. Theoretical HRD for intermediate mass and massive stars of lower mass with composition $Y=0.230$ and $Z=0.0001$. Each evolutionary track goes from the ZAMS to either the start of the TP-AGB phase $\left(M \leq M_{\text {up }}\right)$ or the stage of core C-ignition $\left(M \geq M_{\mathrm{up}}\right)$. These models are calculate with core and envelope overshoot $\left(\Lambda_{\mathrm{c}}=0.5\right.$ and $\Lambda_{\mathrm{e}}=0.7$, respectively). The massive stars in the range $M_{\text {up }} \leq M \leq 9 M_{\odot}$ are calculated at constant mass. The mass of the star in solar unit is indicated along each curve

mass loss by stellar wind can be neglected. The models go from the ZAMS to the latest evolutionary phase, i.e. TPAGB for stars up to $M_{\text {up }}$ and central C-ignition above. The value of $M_{\text {up }}$ falls between $4.0 M_{\odot}$ and $5.0 M_{\odot}$. The more impressive features exhibited by these tracks are the extended loops on the HRD of the He-burning sequences.

Figure 7 shows the models of massive stars in which the effect of mass loss all over their evolution is important. Each sequence goes from the ZAMS to the stage of central C-ignition. Relevant is the dependence of massloss rate on metallicity which strongly affects both the morphology of the HRD and the lifetimes of the various evolutionary phases. In particular these metal-poor models of any initial mass start burning helium early in their way to the red supergiant phase, and are found to never enter the Wolf-Rayet stages.

Table 1 contains the lifetimes of the central $\mathrm{H}$ - and Heburning phases and early AGB (E-AGB) of all the models. The three lifetimes are indicated by $\tau_{\mathrm{H}}, \tau_{\mathrm{He}}$, and $\tau_{\mathrm{E}-\mathrm{AGB}}$, respectively.

Table 2 summarizes the changes in the surface abundances (by mass) induced by the first dredge-up (the pollution of the original surface composition by the products of H-burning) as function of the stellar mass. For 
Table 1. Lifetimes and lifetime ratios

\begin{tabular}{ccccc}
\hline$M / M_{\odot}$ & $\tau_{\mathrm{H}}$ & $\tau_{\mathrm{He}}$ & $\tau_{\mathrm{He}} / \tau_{\mathrm{H}}$ & $\tau_{\mathrm{E}-\mathrm{AGB}} / \tau_{\mathrm{He}}$ \\
\hline 0.51 & $\ldots$ & $1.230 \mathrm{e} 8$ & $\ldots$ & 0.078 \\
0.53 & $\ldots$ & $1.176 \mathrm{e} 8$ & $\ldots$ & 0.080 \\
0.55 & $\ldots$ & $1.144 \mathrm{e} 8$ & $\ldots$ & 0.081 \\
0.57 & $\ldots$ & $1.119 \mathrm{e} 8$ & $\ldots$ & 0.080 \\
0.60 & $3.739 \mathrm{e} 10$ & $1.075 \mathrm{e} 8$ & 0.003 & 0.083 \\
0.65 & $\ldots$ & $1.122 \mathrm{e} 8$ & $\ldots$ & 0.069 \\
0.70 & $2.102 \mathrm{e} 10$ & $1.008 \mathrm{e} 8$ & 0.005 & 0.083 \\
0.75 & $\ldots$ & $9.858 \mathrm{e} 7$ & $\ldots$ & 0.081 \\
0.80 & $1.266 \mathrm{e} 10$ & $9.796 \mathrm{e} 7$ & 0.008 & 0.078 \\
0.90 & $8.117 \mathrm{e} 9$ & $9.655 \mathrm{e} 7$ & 0.012 & 0.076 \\
1.0 & $5.480 \mathrm{e} 9$ & $9.518 \mathrm{e} 7$ & 0.017 & 0.074 \\
1.1 & $3.880 \mathrm{e} 9$ & $9.421 \mathrm{e} 7$ & 0.024 & 0.069 \\
1.2 & $2.842 \mathrm{e} 9$ & $9.462 \mathrm{e} 7$ & 0.033 & 0.065 \\
1.3 & $2.139 \mathrm{e} 9$ & $9.395 \mathrm{e} 7$ & 0.044 & 0.062 \\
1.4 & $1.655 \mathrm{e} 9$ & $9.297 \mathrm{e} 7$ & 0.056 & 0.060 \\
1.5 & $1.461 \mathrm{e} 9$ & $9.504 \mathrm{e} 7$ & 0.065 & 0.056 \\
1.6 & $1.199 \mathrm{e} 9$ & $1.002 \mathrm{e} 8$ & 0.084 & 0.053 \\
1.7 & $1.002 \mathrm{e} 9$ & $1.540 \mathrm{e} 8$ & 0.154 & 0.038 \\
1.8 & $8.494 \mathrm{e} 8$ & $1.678 \mathrm{e} 8$ & 0.198 & 0.030 \\
1.9 & $7.299 \mathrm{e} 8$ & $1.497 \mathrm{e} 8$ & 0.205 & 0.030 \\
2.0 & $6.350 \mathrm{e} 8$ & $1.301 \mathrm{e} 8$ & 0.05 & 0.030 \\
2.5 & $3.667 \mathrm{e} 8$ & $6.441 \mathrm{e} 7$ & 0.176 & 0.033 \\
3.0 & $2.466 \mathrm{e} 8$ & $3.742 \mathrm{e} 7$ & 0.152 & 0.036 \\
4.0 & $1.379 \mathrm{e} 8$ & $1.651 \mathrm{e} 7$ & 0.120 & 0.035 \\
5.0 & $9.007 \mathrm{e} 7$ & $9.126 \mathrm{e} 6$ & 0.101 & 0.038 \\
6.0 & $6.437 \mathrm{e} 7$ & $5.780 \mathrm{e} 6$ & 0.090 & 0.035 \\
7.0 & $4.849 \mathrm{e} 7$ & $3.967 \mathrm{e} 6$ & 0.082 & 0.033 \\
9.0 & $3.147 \mathrm{e} 7$ & $2.272 \mathrm{e} 6$ & 0.072 & 0.026 \\
12.0 & $1.985 \mathrm{e} 7$ & $1.343 \mathrm{e} 6$ & 0.068 & 0.024 \\
15.0 & $1.438 \mathrm{e} 7$ & $9.480 \mathrm{e} 5$ & 0.066 & 0.022 \\
20.0 & $1.001 \mathrm{e} 7$ & $6.580 \mathrm{e} 5$ & 0.066 & 0.020 \\
30.0 & $6.544 \mathrm{e} 6$ & $4.648 \mathrm{e} 5$ & 0.071 & 0.014 \\
40.0 & $5.138 \mathrm{e} 6$ & $3.919 \mathrm{e} 5$ & 0.076 & 0.012 \\
\hline & & & &
\end{tabular}

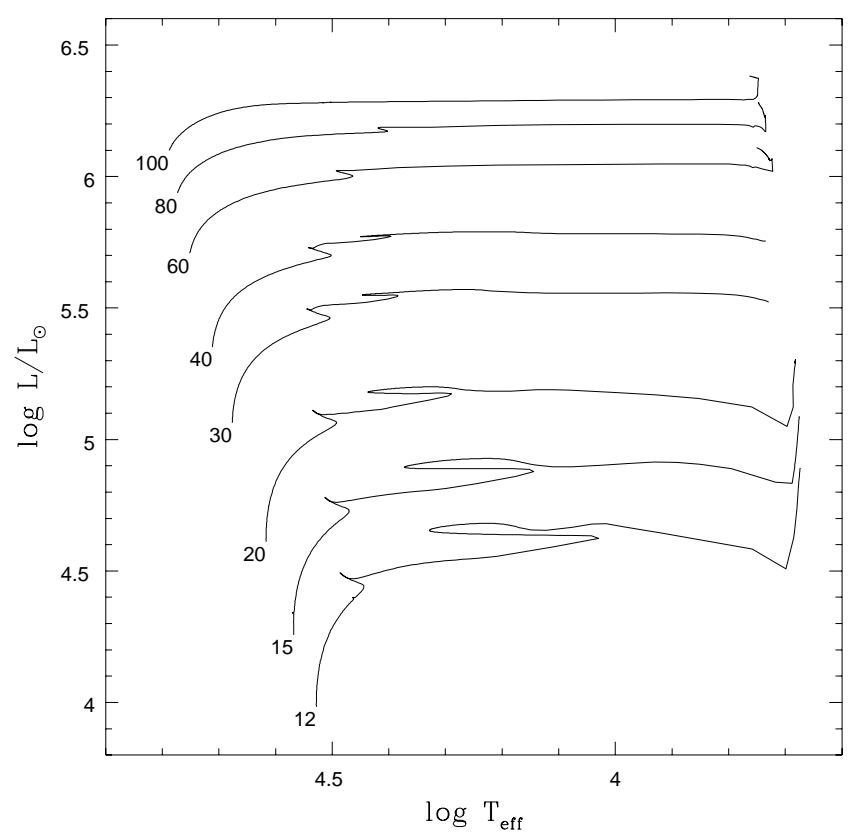

Fig. 7. Theoretical HRD for massive stars with composition $Y=0.230$ and $Z=0.0001$ calculated with core and envelope overshoot, and mass loss by stellar winds. Each evolutionary track goes from the ZAMS to the stage of core C-ignition. The parameters for core and envelope overshoot are $\Lambda_{\mathrm{c}}=0.5$ and $\Lambda_{\mathrm{e}}=0.7$, respectively

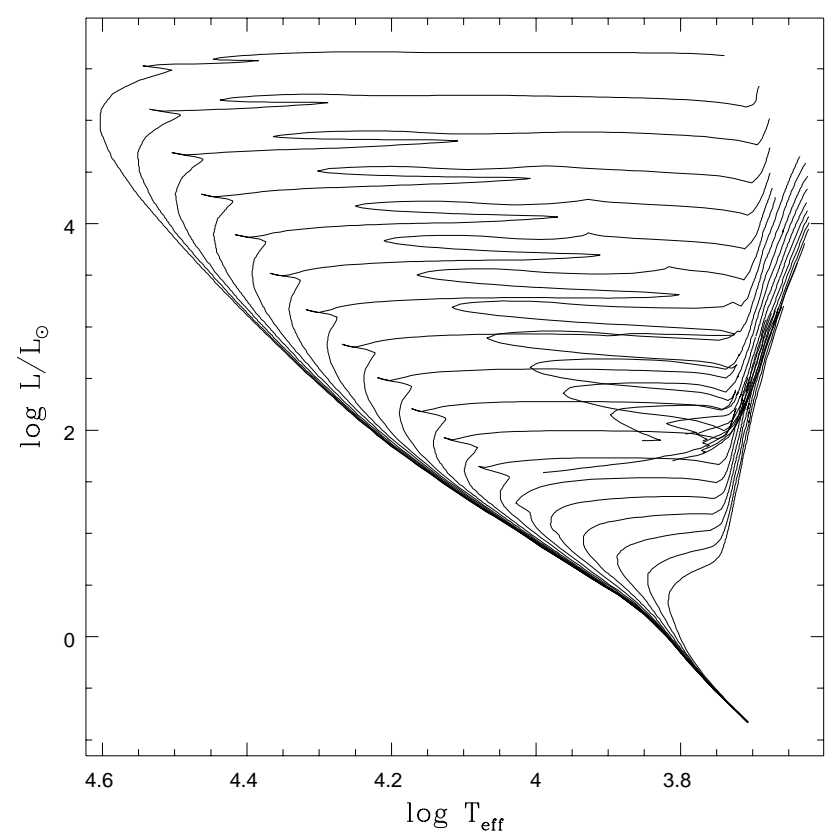

Fig. 8. HRD for a subset of the theoretical isochrones derived from the tracks described in this paper. Ages are from $\log ($ age $/ \mathrm{yr})=6.8$ to 10.2 at intervals of 0.2 . The lower end of all the isochrones corresponds to the MS model of $0.6 M_{\odot}$. For the sake of clarity, we do not plot the portion of the isochrones corresponding to the post-AGB sequences

the elements ${ }^{1} \mathrm{H},{ }^{3} \mathrm{He},{ }^{4} \mathrm{He},{ }^{12} \mathrm{C},{ }^{13} \mathrm{C},{ }^{14} \mathrm{~N},{ }^{15} \mathrm{~N},{ }^{16} \mathrm{O},{ }^{17} \mathrm{O}$, and ${ }^{18} \mathrm{O}$, this table displays both their initial abundance and their surface abundance after completion of the first dredge-up. The third column gives the fractionary mass $\left(Q_{\text {conv }}\right)$ of the layer reached by the external convection at its maximum penetration, while the last two columns show the ratios ${ }^{12} \mathrm{C} /{ }^{13} \mathrm{C}$ and $\left({ }^{14} \mathrm{~N}\right) /\left({ }^{14} \mathrm{~N}\right)_{\mathrm{i}}$. This latter ratio is the enhancement factor of ${ }^{14} \mathrm{~N}$ with respect to the initial value $\left({ }^{14} \mathrm{~N}\right)_{\mathrm{i}}$.

For each star Table 3 indicates the age at which the second dredge-up occurs (when the external convection reaches the deep He-rich layers between the inert carbonoxygen core and the H-burning shell temporarily extinguished), the mass $Q_{\text {conv }}$ of the layer reached by the external convection at its maximum penetration, and the surface abundances (by mass) of ${ }^{1} \mathrm{H},{ }^{4} \mathrm{He},{ }^{12} \mathrm{C},{ }^{13} \mathrm{C},{ }^{14} \mathrm{~N}$ and ${ }^{16} \mathrm{O}$.

Remarkably, models with mass higher than $3 M_{\odot}$ do not become red giants before the start of He-burning, and consequently they miss any dredge-up event before entering on the E-AGB. Moreover, when the second dredgeup occurs on these stars, C-burning starts on the interior when the external convection has not yet attained its maximum extension. Consequently, the surface abundance continuously varies during their evolution along the Hayashi line. 
Table 2. Surface abundances after the $1^{\text {st }}$ dredge-up

\begin{tabular}{|c|c|c|c|c|c|c|c|c|c|c|c|c|c|c|}
\hline$M / M_{\odot}$ & Age & $Q_{\text {eonv }}$ & ${ }^{1} \mathbf{H}$ & ${ }^{3} \mathrm{He}$ & ${ }^{4} \mathrm{He}$ & ${ }^{12} \mathrm{C}$ & ${ }^{19} \mathrm{C}$ & $11 \mathrm{~N}$ & ${ }^{15} \mathrm{~N}$ & 160 & 170 & $\operatorname{la}^{a} 0$ & ${ }^{12} \mathrm{C} /{ }^{13} \mathrm{C}$ & ${ }^{14} \mathrm{~N} /\left({ }^{14} \mathrm{~N}\right)_{\mathrm{i}}$ \\
\hline \multicolumn{3}{|c|}{ Initial values } & 0.7699 & $2,46 k-5$ & 0.230 & $1.713 e-5$ & $2.065 e-7$ & $5.298 \mathrm{e}-6$ & $2.090 e-8$ & $4.813 \mathrm{e}-5$ & $1.950=-8$ & $1.085 e-7$ & 82.97 & 1.00 \\
\hline 0.60 & $3.939=10$ & 0.513 & 0.765 & $2.758 e-3$ & 0.232 & $1.713 \mathrm{e}-5$ & $2.070 \mathrm{e}-7$ & $5.298 e-6$ & $2.089 \mathrm{e}-8$ & $4.813 e-5$ & $1.950<-8$ & $1.085 e-7$ & 82.78 & 1.00 \\
\hline 0.70 & $2.233 \mathrm{e} 10$ & 0.451 & 0.762 & 2.077 e-3 & 0.236 & $1.710 \mathrm{e}-5$ & $2.430=-7$ & $5.301 e-6$ & $2.001<-8$ & $4,813 \mathrm{e}-5$ & $1.950 \mathrm{e}-8$ & $1.085 e-7$ & 70.37 & 1.00 \\
\hline 0.80 & $1.358 \mathrm{e} 10$ & 0.403 & 0.760 & $1.562 \mathrm{e}-3$ & 0.239 & $1.6800-5$ & $4.175 e-7$ & $5.462 e-6$ & 1.830 e- 8 & $4.813 \mathrm{e}-5$ & $1.951 \mathrm{e}-8$ & 1.0B3e-7 & 40.25 & 1.03 \\
\hline 0.90 & B.804e9 & 0.365 & 0.758 & $1.235 e-3$ & 0.241 & $1.594 \mathrm{e}-5$ & $4.969=-7$ & $6.388 \mathrm{e}-6$ & $1.695 \mathrm{e}-8$ & $4.813 \mathrm{e}-5$ & $1.960 \mathrm{c}-8$ & $1.067 \mathrm{e}-7$ & 32.07 & 1.21 \\
\hline 1.00 & $6.01 \% \mathrm{eg}$ & 0.336 & 0.756 & g.861e-4 & 0.243 & $1.504 \mathrm{e}-5$ & $5.210 \mathrm{e}-7$ & $7.408-6$ & $1.565 e-8$ & $4.813 \mathrm{e}-5$ & $2.033 \mathrm{e}-\mathrm{B}$ & $1.027 \mathrm{e}-7$ & 28.87 & 1.40 \\
\hline 1.10 & $4.292 \mathrm{eg}$ & 0.312 & 0.755 & $8.163 e-4$ & 0.244 & $1.444 \mathrm{e}-5$ & $5.377 e-7$ & $8.096 e-6$ & $1.487 \mathrm{e}-8$ & $4.813 e-5$ & $2.363 c-8$ & $9.950 \mathrm{e}-\mathrm{B}$ & 26.86 & 1.53 \\
\hline 1.20 & 3.173e9 & 0.295 & 0.754 & $6.897 e-4$ & 0.246 & $1.378 \mathrm{e}-5$ & $5.298 \mathrm{e}-7$ & 8.878-6 & $1.408 e-8$ & $4,812 e-5$ & 3.429e-8 & $9.570 \mathrm{e}-\mathrm{B}$ & 26.01 & 1.68 \\
\hline 1.30 & $2.424 \mathrm{eg}$ & 0.271 & 0.753 & $5,824 e-4$ & 0.248 & $1.317 e-5$ & $8.445 e-7$ & $9,581 \subset-6$ & $1.327 e-8$ & $4.8100-5$ & $3.936 e-8$ & $9.236 \mathrm{e}-8$ & 24.18 & 1.81 \\
\hline 1.40 & $1.875 e 9$ & 0.272 & 0.753 & $5.053 c-4$ & 0.246 & $1.271 \mathrm{e}-5$ & $5.494 e-7$ & $1.0110-5$ & $1.266 \mathrm{e}-8$ & $4.805 e-5$ & $1.031 \mathrm{e}-7$ & $9.014 e-8$ & 23.14 & 1.91 \\
\hline 1.50 & $1.356 e 9$ & 0.246 & 0.753 & $4.630=-4$ & 0.246 & $1.239 e-5$ & $8.6340-7$ & $1.047 e-5$ & $1.217 \mathrm{e}-8$ & $4.8040-5$ & $1.142 \mathrm{e}-7$ & 8.794e-8 & 21.99 & 1.98 \\
\hline 1.60 & $1.266 \mathrm{e} 9$ & 0.234 & 0.754 & $4,068 c-4$ & 0.246 & $1.1900-5$ & $5.755 e-7$ & $1.095-5$ & $1.166 \mathrm{e}-8$ & $4.795 e-5$ & $1.909<-7$ & $8.569=-8$ & 20.83 & 2.07 \\
\hline 1.70 & $1.050 \mathrm{eg}$ & 0.225 & 0.753 & $3.631 e-4$ & 0.246 & $1.148 \mathrm{e}-5$ & $5.475 e-7$ & $1.162 \mathrm{e}-5$ & $1.124 \mathrm{e}-8$ & $4.779 \mathrm{e}-5$ & 2.943e-7 & $8.259 \mathrm{e}-\mathrm{B}$ & 20.98 & 2.19 \\
\hline 1.80 & 8.820e8 & 0.223 & 0.754 & $3.270 c-4$ & 0.246 & $1.125 e-5$ & $5.508 e-7$ & $1.195 e-5$ & $1.085 e-8$ & $4.765-5$ & $3.757 e-7$ & $8.105 e-8$ & 20.42 & 2.26 \\
\hline 1.90 & $7,502 \mathrm{eg}$ & 0.246 & 0.756 & $3.081 e-4$ & 0.243 & $1.143 e-5$ & $5.858 \mathrm{e}-7$ & $1.165-5$ & $1.087 \mathrm{c}-8$ & $4.770 \mathrm{e}-5$ & $3.908 e-7$ & $8.233 e-8$ & 19.51 & 2.20 \\
\hline 2.00 & $6.495 \mathrm{eB}$ & 0.263 & 0.759 & $2.872 \mathrm{e}-4$ & 0.241 & $1.146 \mathrm{e}-5$ & $5.235 e-7$ & $1.164 \mathrm{e}-5$ & $1.114 e^{-8}$ & $4.7 \mathrm{B3e-5}$ & $3.046 e-7$ & $8.164 e-8$ & 21.88 & 2.20 \\
\hline 2.50 & $3.714 \mathrm{eB}$ & 0.417 & 0.767 & $2.1510-4$ & 0.233 & $1.236 \mathrm{e}-5$ & $6.476 e-7$ & $1.042-5$ & $1.148 \mathrm{e}-8$ & $4.812 \mathrm{e}-5$ & 3.825e-8 & $9.044 \mathrm{e}-8$ & 19.08 & 1.97 \\
\hline 3.00 & $2.489 \mathrm{eg}$ & 0.563 & 0.769 & $1.754 e-4$ & 0.231 & $1.403 \mathrm{e}-5$ & $7.227 \mathrm{e}-7$ & $8.372 e-6$ & $1.304 \mathrm{e}-8$ & $4.813 \mathrm{e}-5$ & $2.033 e-8$ & $1.022 e-7$ & 19.42 & 1.58 \\
\hline
\end{tabular}

Table 3. Surface abundances after the $2^{\text {nd }}$ dredge-up

\begin{tabular}{ccccccccc}
\hline \hline$M / M_{\odot}$ & Age & $Q_{\text {conv }}$ & ${ }^{1} \mathrm{H}$ & ${ }^{4} \mathrm{He}$ & ${ }^{12} \mathrm{C}$ & ${ }^{13} \mathrm{C}$ & ${ }^{14} \mathrm{~N}$ & $16 \mathrm{O}$ \\
\hline 1.80 & $1.055 \mathrm{e} 9$ & 0.324 & 0.753 & 0.246 & $1.022 \mathrm{E}-05$ & $4.699 \mathrm{E}-05$ & $1.365 \mathrm{E}-05$ & $5.748 \mathrm{E}-07$ \\
1.90 & $9.048 \mathrm{e} 8$ & 0.313 & 0.756 & 0.244 & $1.031 \mathrm{E}-05$ & $4.691 \mathrm{E}-05$ & $1.357 \mathrm{E}-05$ & $6.107 \mathrm{E}-07$ \\
2.00 & $7.837 \mathrm{e} 8$ & 0.308 & 0.758 & 0.241 & $1.027 \mathrm{E}-04$ & $4.696 \mathrm{E}-05$ & $1.370 \mathrm{E}-05$ & $5.569 \mathrm{E}-07$ \\
2.50 & $4.379 \mathrm{e} 8$ & 0.289 & 0.761 & 0.239 & $1.048 \mathrm{E}-05$ & $4.650 \mathrm{E}-05$ & $1.390 \mathrm{E}-05$ & $5.648 \mathrm{E}-07$ \\
3.00 & $2.876 \mathrm{e} 8$ & 0.272 & 0.751 & 0.249 & $9.233 \mathrm{E}-06$ & $4.525 \mathrm{E}-05$ & $1.638 \mathrm{E}-05$ & $4.912 \mathrm{E}-07$ \\
\hline
\end{tabular}

In massive stars, the variation of the surface abundances in the course of evolution is not as simple as in the lower mass range, but it is complicated by the occurrence of mass loss and depends also on the efficiency of central mixing in the sense that the larger the convective core, the easier is the exhibition of nuclearly processed material at the surface.

\section{Theoretical isochrones}

Theoretical isochrones were constructed from the tracks presented in this paper. A detailed description of the methods is presented in Bertelli et al. (1990, 1994). Below we just recall some aspects of the construction of these isochrones.

To include in the isochrones the effects of mass loss by stellar wind towards the tip of the RGB for low mass stars and during the AGB evolution of low and intermediate mass stars, the empirical formulation by Reimers (1975) is used. The mass loss parameter $\eta$ varies from 0.35 for low mass stars increasing gradually to $\eta=1$ for intermediate mass stars. In passing from the tip of the RGB to the ZAHB, we integrate the mass loss rate along the RGB to estimate the total amount of mass that has to be removed. Then the mass of the evolutionary models (that were computed at constant mass) is simply scaled down to the value suited to the ZAHB stars, as the effects of mass loss on the internal structure of models at the tip of the RGB are negligible.

Mass loss is the key parameter during the evolution in the TP-AGB, while it can be neglected during the early AGB phase. From the beginning of the TP-AGB phase up to the stage of envelope ejection, the evolutionary tracks are evolved analytically (see Renzini 1977; Iben \& Renzini 1983; Bertelli et al. 1990, 1994; Grönewegen \& de Jong 1993 for details). Like in Bertelli et al. (1994), we adopt the core mass-luminosity relationship from the models by Boothroyd \& Sackmann (1988), Reimers' mass loss rate with the parameter $\eta$ increasing with the stellar mass, and a luminosity-effective temperature relation obtained extrapolating the slope of the early AGB phase of our models to higher luminosities and lower effective temperatures. The tracks are ended when the entire mass of the envelope is removed.

Therefore, the terminal stage of the isochrones is either a white dwarf for $M_{\mathrm{i}} \leq 4 M_{\odot}$, or core C-ignition for the remaining stars.

When the star evolves toward the white dwarf stage, the isochrones include also the evolutionary phase across the HRD as the central star of a planetary nebula (CSPN). In this case we derive a suitable relation between the initial mass and the mass of the CSPN. The evolutionary sequences for the CSPNs are either taken from Schönberner (1983) and Blöcker \& Schönberner (1990) for $M_{\mathrm{cs}}=0.546$, 


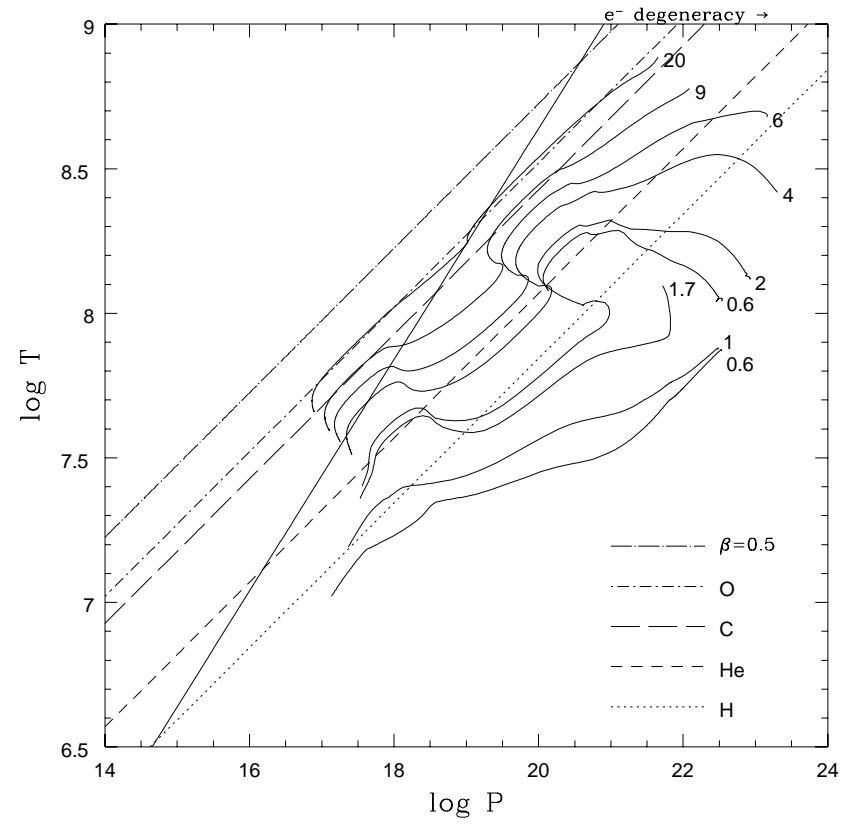

Fig. 9. In the plane $(P, T)$, we limit the regions in which our EOS change its characteristics. Going to higher pressures at constant temperatures: $\beta=0.5$ limits the region in which gas pressure begins to dominate over radiation pressure. The lines labelled with $\mathrm{O}, \mathrm{C}, \mathrm{He}$ and $\mathrm{H}$ limit the areas in which $P_{\mathrm{c}}$ becomes $1 \%$ of the gas pressure for, respectively, pure $\mathrm{O}, \mathrm{C}$, $\mathrm{He}$ and $\mathrm{H}$ compositions. We use Eq. (A12) to determine this limit for any chemical mixture. The continuous straight line limits the region in which we start considering electron degeneracy. Superimposed, we see the run of central temperature and pressure for some stars of different masses (this paper)

$0.565,0.605$ and $0.836 M_{\odot}$ or explicitly calculated for $M_{\mathrm{cs}}=0.5167$ and $0.646 M_{\odot}$ (see Bertelli et al. 1994). All these models possess the same initial chemical composition, namely $[Z=0.020, Y=0.28]$ and have the same initial stage taken at $\log T_{\text {eff }}=3.70$ (the short-lived part of the sequence between the tip of the AGB and this initial stage is neglected). The age of the stellar models in the CSPN stage is inclusive of the lifetime elapsed from the zero age main sequence up to the tip of the AGB.

Theoretical luminosities and effective temperatures along the isochrones are translated to magnitudes and colors using extensive tabulations of bolometric corrections and colors obtained from properly convolving the spectral energy distributions contained in the library of stellar spectra kindly made available by Kurucz (1992). The response functions for the various pass-bands in which magnitudes and colors are generated are from the following sources: Buser \& Kurucz (1978) for the $U B V$ passbands, Bessell (1990) for the $R$ and $I$ Cousins pass-bands, and finally Bessell \& Brett (1988) for the $J H K$ pass-bands.

In Fig. 8 we present some of the isochrones on the HRD, while Table 4 presents a summary of the most significant stages of the isochrones, sampled at age intervals of $\Delta \log t=0.1$ (ages are in years). The characteristic stages are:

a) The turnoff (TO), i.e. the bluest point during core $\mathrm{H}$ burning.

b) The reddest point before the overall contraction phase at the end of the core H-burning (indicated as stage B).

c) The stage of core $\mathrm{H}$-exhaustion (indicated as stage $\mathrm{C}$ ).

d) The base of the RGB ( $\left.B_{\mathrm{RGB}}\right)$.

e) The tip of the RGB ( $\left.T_{\mathrm{RGB}}\right)$.

f) The mean locus of the core-He burning phase $\left(M_{\mathrm{Heb}}\right)$. This is evaluated graphically, plotting the luminosity (or $M_{V}$ ) versus the current mass along the isochrone.

g) The bluest stage during core He-burning in presence of a loop $\left(B_{\mathrm{Heb}}\right)$.

h) The reddest stage during core He-burning in presence of a loop $\left(R_{\mathrm{Heb}}\right)$.

i) The tip of the AGB $\left(T_{\mathrm{AGB}}\right)$.

j) Finally, the last computed model (LM) for all cases in which the AGB phase does not occur.

The layout of Table 4 is as follows: Column 1 (Log(age)): the logarithm of the age in yr; Column 2 (Phase): the characteristic stage; Column $3\left(M / M_{\odot}\right)$ : the current mass in solar units; Column $4\left(\log T_{\text {eff }}\right)$ : the logarithm of the effective temperature; Column $5\left(\log L / L_{\odot}\right)$ the logarithm of the luminosity in solar units; Column 6 $\left(M_{V}\right)$ : the absolute visual magnitude; Columns 7-10: the colors $(B-V),(V-I),(V-J)$, and $(V-K)$, respectively.

Complete tables with the isochrones are available on request, and can also be obtained through the WWW (see Sect. 4). These tables are specially useful in the study of very metal poor populations as those found on halo globular clusters and some dwarf spheroidal galaxies.

\section{Summary and conclusions}

We have presented a new grid of evolutionary tracks and isochrones with extremely low metallicity. They cover a wide range of evolutionary stages and masses and are suitable for population synthesis purposes. They complement those by Bertelli et al. (1994a and references therein) at the low-metallicity end.

Results and peculiar features of the models are briefly commented. We discuss the main differences that arise on these models due to the adoption of some different physical ingredients. Particularly, we evaluate the change in the lifetimes of the models as a function of stellar mass, due to the introduction of Coulomb interactions on the EOS for the stellar interior. The tracks described by the models on the HRD do not change significantly due to the introduction of these different ingredients.

These grids of models belong to a wider data base of stellar models aimed at studying the spectro-photometric evolution of stellar populations of different complexity going from star clusters to galaxies. Most of this data base has already been published in the previous papers of this series. 
Acknowledgements. We thank Dr. O. Straniero for his help in clarifying some of the aspects concerning the Coulomb corrections. This work has been financially supported by the Italian Ministry of University, Scientific Research and Technology (MURST) and the Italian Space Agency (ASI). L. Girardi acknowledges a fellowship from Brazilian funding agency CNPq.

\section{A. Appendix: The interior EOS}

In a dense plasma the EOS deviates from that of an ideal gas due to the effect of the Coulomb interactions between the charged particles. Due to the tendency of electrons to cluster around ions, the net effect is that a negative term is added to the pressure and internal energy of the stellar material at these conditions.

We refer to Straniero (1988) for a general description of the subject. Here we limit to a description of the formulas adopted by ourselves, and a summary analysis of the effects of the corrections in our models.

We consider the total pressure $P$, and the energy by unit mass of stellar material $u$ given as a function of density $\rho$ and temperature $T$ by

$$
\begin{aligned}
P & =\left(\frac{N_{\mathrm{a}} k}{\mu_{0}} \rho T+P_{\mathrm{e}}\right)+\frac{a T^{4}}{3}+P_{\mathrm{c}}(\rho, T) \\
u & =\left(\frac{3 \mathcal{R}}{2 \mu_{0}}+u_{\mathrm{e}}\right)+\frac{a T^{4}}{\rho}+u_{\mathrm{c}}(\rho, T)
\end{aligned}
$$

The right-hand side terms refer respectively to the contributions of kinetic energy, radiation, and the Coulomb interactions between all gas particles. Symbols have their usual meaning, namely: $\mu_{0}$ is the mean atomic weight, $N_{\mathrm{a}}$ is Avogadro's number, $k$ is Boltzmann's constant, $a$ is the radiation pressure constant. The subscript e stands for the electrons. $u_{\mathrm{c}}$ is related to $P_{\mathrm{c}}$ by $u_{\mathrm{c}}=3 P_{\mathrm{c}} / \rho$.

As usual, the parameter which describes the magnitude of the Coulomb energy is $\Gamma$, which is a ratio between the mean kinetic and potential energy of ions. For a onecomponent neutral plasma, $\Gamma=(Z e)^{2} /\langle r\rangle k T$, with $Z$ and $A$ being respectively the charge and atomic mass of ions, and $\langle r\rangle=\left(4 \pi \rho N_{\mathrm{a}} / 3 A\right)^{-1 / 3}$ the mean distance between ions. The Coulomb energy for the ions, $u_{\mathrm{ci}}$, is then a simple function of $\Gamma$, being expressed by a fraction $f(\Gamma)$ of the kinetic energy of ions:

$$
u_{\mathrm{ci}}=N_{\mathrm{a}} k T f(\Gamma) / \mu_{0}
$$

(see Straniero 1988).

There are analytical formulas for $f(\Gamma)$ which fit both the results of Debye-Hückel theory at low $\Gamma$ and the results of Monte Carlo simulations at high $\Gamma$ (e.g. Hansen 1973; Hansen \& Vieillefosse 1976). To deal with the Coulomb correction for arbitrary chemical mixtures, we generalize the expressions given by Hansen \& Vieillefosse (1976) for a two-component plasma. Accordingly, for an arbitrary mixture of ions labelled $z$, of electric charge $Z_{z}$, atomic mass $A_{z}$, at mass fractions $X_{z}$, we have:

$$
f(\Gamma)=\mu_{0} \sum_{z} \frac{X_{z}}{A_{z}} f\left(\Gamma_{z}\right)
$$

with

$$
\begin{gathered}
f\left(\Gamma_{z}\right)=\Gamma_{z}^{3 / 2}\left[\frac{-0.900}{\left(0.7025+\Gamma_{z}\right)^{1 / 2}}+\frac{0.274}{1.3195+\Gamma_{z}}\right] \\
\Gamma_{z}=\Gamma^{\prime} Z_{z}^{5 / 3}=\frac{Z^{1 / 3} e^{2}}{\langle r\rangle k T} Z_{z}^{5 / 3}
\end{gathered}
$$

In the latter equation,

$$
\langle r\rangle=\left(\frac{4 \pi \rho N_{\mathrm{a}}}{3 \mu_{0}}\right)^{-1 / 3}
$$

is the mean distance between ions, and

$$
Z^{\prime}=\frac{\mu_{0}}{\sum_{z} Z_{z} X_{z} / A_{z}}=\frac{\mu_{0}}{\mu_{\mathrm{e}}}
$$

is the mean charge of the ions.

The above formulas are identical to those given by Hansen \& Vieillefosse (1976) for two-component plasmas, and are expected to be good approximations for 3 or 4element mixtures. Therefore, are fully adequate to describe the Coulomb energy of ions in the interior of stars at $\mathrm{H}$ and He-burning stages.

The Coulomb energy of the electrons is, however, much more uncertain. For a one-component plasma in the limit of low- $\Gamma$, Straniero's (1988) Eq. (45) gives us an energy by unit mass which is a factor

$$
\frac{u_{\mathrm{c}}}{u_{\mathrm{ci}}}=\frac{Z+1}{Z^{3 / 2}}\left(Z+\frac{F_{1 / 2}(\alpha)_{\psi}}{F_{1 / 2}(\alpha)}\right)^{1 / 2}
$$

greater than that would be obtained simply by the ions (being $\alpha=k T / m_{\mathrm{e}} c^{2}, F_{1 / 2}(\alpha)$ the known Fermi-Dirac integral, and $F_{1 / 2}(\alpha)_{\psi}$ its derivative with respect to the degeneracy parameter $\psi$ ). It is attractive to use this result in order to obtain the total (ions+electrons) Coulomb energy once the ionic contribution is obtained from Eq. (A3), for all values of $\Gamma$.

Using the same simple reasoning that led us to generalize Hansen \& Vieillefosse (1976) formulas to Eqs. (A4 - A8), with the aid of Eq. (A8) we transformed Eq. (A9) into

$$
\frac{u_{\mathrm{c}}}{u_{\mathrm{ci}}}=\left(1+\frac{\mu_{\mathrm{e}}}{\mu_{0}}\right)\left(1+\frac{\mu_{\mathrm{e}}}{\mu_{0}} \frac{F_{1 / 2}(\alpha) \psi}{F_{1 / 2}(\alpha)}\right)^{1 / 2} .
$$

This latter was actually used for the multiple-component plasmas dealt with by our stellar evolution code.

The Coulomb terms to the pressure and internal energy were included in the derivation of all thermodynamical quantities (and their derivatives) of our stellar code. 
Calculation of these terms is accomplished only when the Coulomb pressure overcomes a threshold fraction $\chi$ (set to $1 \%$ ) of the total gas pressure. This condition can be shown to translate into:

$$
\begin{aligned}
& \log P \gtrsim 4 \log T-2 \log \\
& {\left[\mu_{\mathrm{e}}\left(X+1.414 Y+7.348 X_{\mathrm{C}}+11.31 X_{\mathrm{O}}\right)\right]} \\
& +2 \log \chi-7.381 .
\end{aligned}
$$

Figure 9 shows in the plane $(P, T)$ the limits at which we consider electron degeneracy and the Coulomb corrections in our EOS. Superimposed are the run of central temperature and pressure for some of the stellar models presented in this paper. We refer to Straniero (1988) for plots detailing the magnitude of Coulomb corrections in the different regions of the $(P, T)$ plane.

Figure 9 indicates that Coulomb corrections are not expected to affect models of high-mass stars, as well as the intermediate-mass stars at stages of central $\mathrm{H}$ or $\mathrm{He}$ burning. All of the stars with convective envelopes, however, are slightly affected through the change in $\alpha$ which is needed in order to recalibrate the solar model.

For those stars with a dense degenerate core, at the RGB and AGB evolutionary phases, the main effect of Coulomb interactions comes from the increase in central densities which occurs in order to adjust the stellar profile to the presence of an additional negative pressure term. Due to this adjustment, He-core masses at the He-flash are $1 \%$ lower than in the case Coulomb interactions are neglected.

Low-mass stars at central nuclear burning stages, either at the MS or at the HB, are affected both through the increase in central densities and the decrease in the adiabatic gradient caused by Coulomb interactions. The latter effect can give rise to slightly larger convective cores, specially on the HB. The change in internal structure becomes noticeable for the lowest mass stars, varying significantly their luminosities and temperatures, and hence lifetimes (see Sect. 3).

A word of caution is necessary here: at the limit of high degeneracy, Eq. (A9) gives $u_{\mathrm{c}} / u_{\mathrm{ci}} \rightarrow(Z+1) / Z$, when the expected behaviour is $u_{\mathrm{c}} / u_{\mathrm{ci}} \rightarrow 1$ (i.e. negligible contribution from the electrons to $u_{\mathrm{c}}$ ). It means that in the degenerate He-cores of RGB stars we could be overestimating $u_{\mathrm{c}}$ by a factor of about 1.5 .

In order to evaluate to which extent this uncertainty can affect our results, we evolved low-mass star models using a correction

$$
\frac{u_{\mathrm{c}}}{u_{\mathrm{ci}}}=\left(1+\frac{\mu_{\mathrm{e}}}{\mu_{0}} \frac{F_{1 / 2}(\alpha)_{\psi}}{F_{1 / 2}(\alpha)}\right)^{3 / 2}
$$

instead of Eq. (A10). It gives the expected behaviour at both high and low- $\Gamma$. Such models evolved in a track coincident with that of models evolved according to Eq. (A10), and underwent core He-flash at a core mass only $0.001 M_{\odot}$ higher. This experiment indicates that the main effect of Coulomb interactions comes from the large region of the star with intermediate- $\Gamma$. Therefore, the exact behaviour of the Coulomb corrections at high- $\Gamma$ probably does not involve serious problems for models evolved up to the end of the RGB and E-AGB phases.

\section{References}

Alongi M., Bertelli G., Bressan A., Chiosi C., 1991, A\&A 244, 95

Alongi M., Bertelli G., Bressan A., Chiosi C., Fagotto F., Greggio L., Nasi E., 1993, A\&AS 97, 851

Anders E., Grevesse N., 1989, Geochim. Cosmochim. Acta 53, 197

Baker N., Kippenhahn R., 1962, Z. Astroph. 54, 114

Bertelli G., Bressan A., Chiosi C., 1984, A\&A 130, 279

Bertelli G., Bressan A., Chiosi C., 1985, A\&A 150, 33

Bertelli G., Betto R., Bressan A., Chiosi C., Nasi E., Vallenari A., 1990, A\&AS 85, 845

Bertelli G., Bressan A., Chiosi C., Fagotto F, Nasi E., 1994, A\&AS 106, 275

Bessell M.S., 1990, PASP 102, 1181

Bessell M.S., Brett J.M., 1988, PASP 100, 1134

Bessell M.S., Brett J.M., Scholz M., Wood P.R., 1989, A\&AS 77,1

Bessell M.S., Brett J.M., Scholz M., Wood P.R., 1991, A\&AS 87, 621

Blöcker T., Schönberner D., 1990, A\&A 240, L11

Bressan A., Bertelli G., Chiosi C., 1981, A\&A 102, 25

Bressan A., Fagotto F., Bertelli G., Chiosi C., 1994a, A\&AS 100,647

Bressan A., Chiosi C., Fagotto F., 1994b, ApJS 94, 63

Buser R., Kurucz R.L., 1978, A\&A 70, 555

Caughlan G.R., Fowler W.A., 1988, Atomic Data Nucl. Data Tables 40, 283

Chiosi C., Bertelli G., Bressan A., 1992a, ARA\&A 30, 305

Chiosi C., Bertelli G., Bressan A., 1992b, in Instabilities in Evolved Super and Hypergiants. In: de Jager C. \& Nieuwenhuijzen H. (eds.). Amsterdam: North Holland Publ., p. 145

Chiosi C., Maeder A., 1986, ARA\&A 24, 239

de Jager C., Nieuwenhuijzen H., van der Hucht K.A., 1988, A\&AS 72, 259

Fagotto F., Bressan A., Bertelli G., Chiosi C., 1994a, A\&AS 104,365

Fagotto F., Bressan A., Bertelli G., Chiosi C., 1994b, A\&AS 105, 29

Fagotto F., Bressan A., Bertelli G., Chiosi C., 1994c, A\&AS 105, 39

Girardi L., Chiosi C., Bertelli G., Bressan A., 1995a, A\&A 298, 87

Girardi L., Bressan A., Chiosi C., 1995b, in Stellar Evolution: What Should Be Done, 32nd Liege International Astrophysical Colloquium (in press)

Grevesse N., 1991, A\&A 242, 488

Grevesse N., Noels A., 1993, Phys. Scr. T, 47, 133

Groenewegen M.A.T., de Jong T., 1993, A\&A 267, 410

Hansen J.-P., 1973, Phys. Rev. A8, 3096

Hansen J.-P., Vieillefosse P., 1976, Phys. Rev. Let. 37, 391 
Huebner W.F., Merts A.L., Magee N.H., Argo M.F., 1977, Los Alamos Scientific Laboratory Report LA-6760-M

Iben I., Renzini A., 1983, ARA\&A 21, 27

Iglesias C.A., Rogers F.J., Wilson B.G., 1992, ApJ 397, 717

de Jager C., Nieuwenhuijzen H., van der Hucht K.A., 1988, A\&AS 72, 259

Kippenhahn R., Thomas H.-C., Weigert A., 1965, Z. Astrophys. 61,241

Kudritzki R.P., Pauldrach A., Puls J., Abbot D.C., 1989, A\&A 219, 205

Kurucz R.L., 1992, in Stellar Populations of Galaxies. In: Barbuy B. and Renzini A. (eds.). Dordrecht: Kluwer, p. 225

Landré V., Prantzos N., Aguer P., Bogaert G., Lefebvre A., Thibaud J.P., 1990, A\&A 240, 85
Mihalas D., Hummer D.G., Mihalas B.W., Däppen W., 1990, ApJ 350, 300

Reimers D., 1975, Mem. Soc. R. Sci. Liege, Ser. 6, Vol. 8, p. 369

Renzini A., 1977, in Advanced Stages of Stellar Evolution. In: Bouvier P. and Maeder A. (eds.), Geneva Observatory, p. 151

Rogers F.J., Iglesias C.A., 1992, ApJS 79, 507

Schaerer D., Meynet G., Maeder A., Schaller G., 1993, A\&AS 98,523

Schaller G., Schaerer D., Meynet G., Maeder A., 1992, A\&AS 96, 269

Schönberner D., 1983, ApJ 272, 708

Straniero O., 1988, A\&AS 76, 157

Weaver T.A., Woosley S.E., 1993, Phys. Rep. 227, 65 
Table 4. Characteristics stages in the isochrones

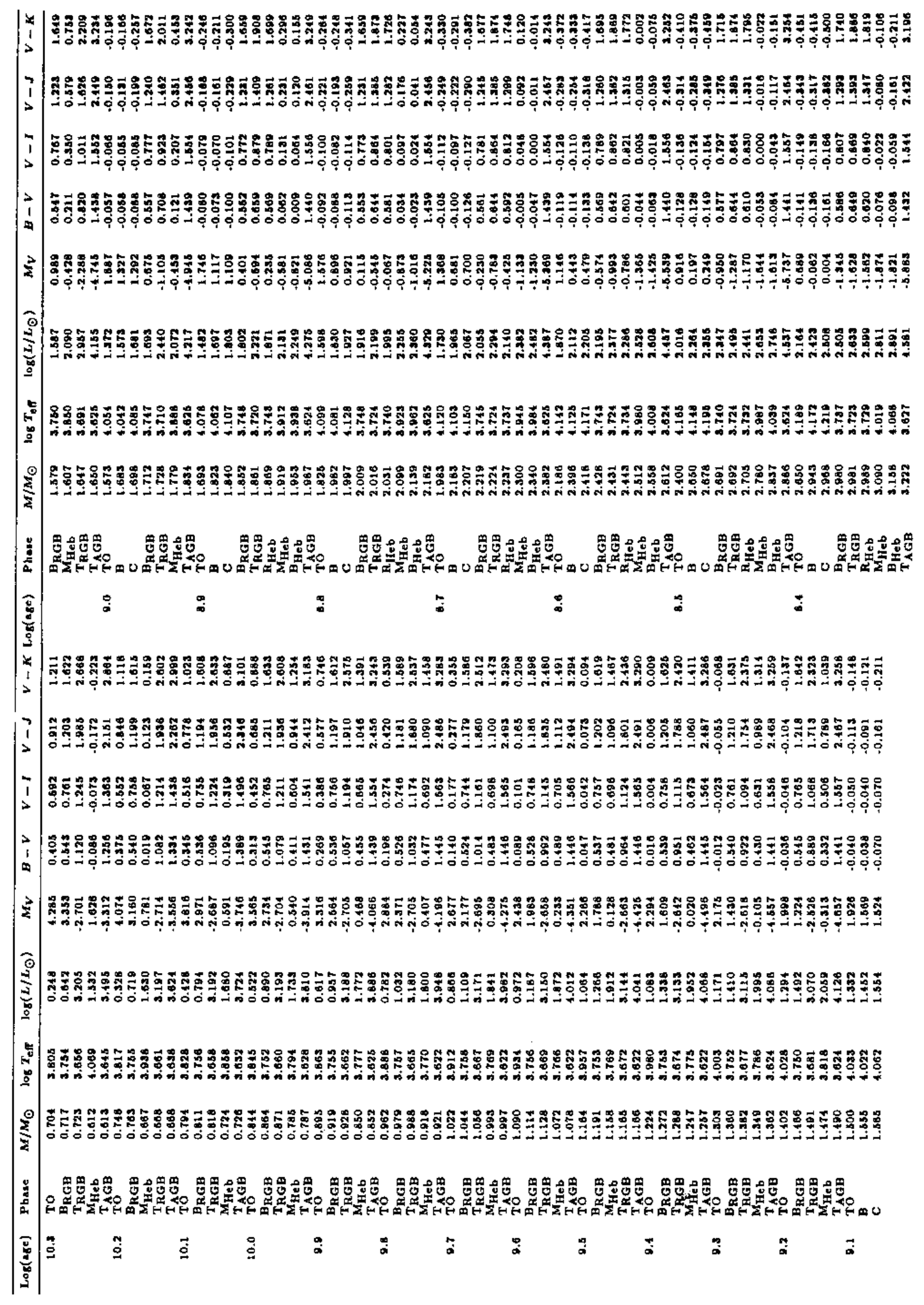


Table 4. continued

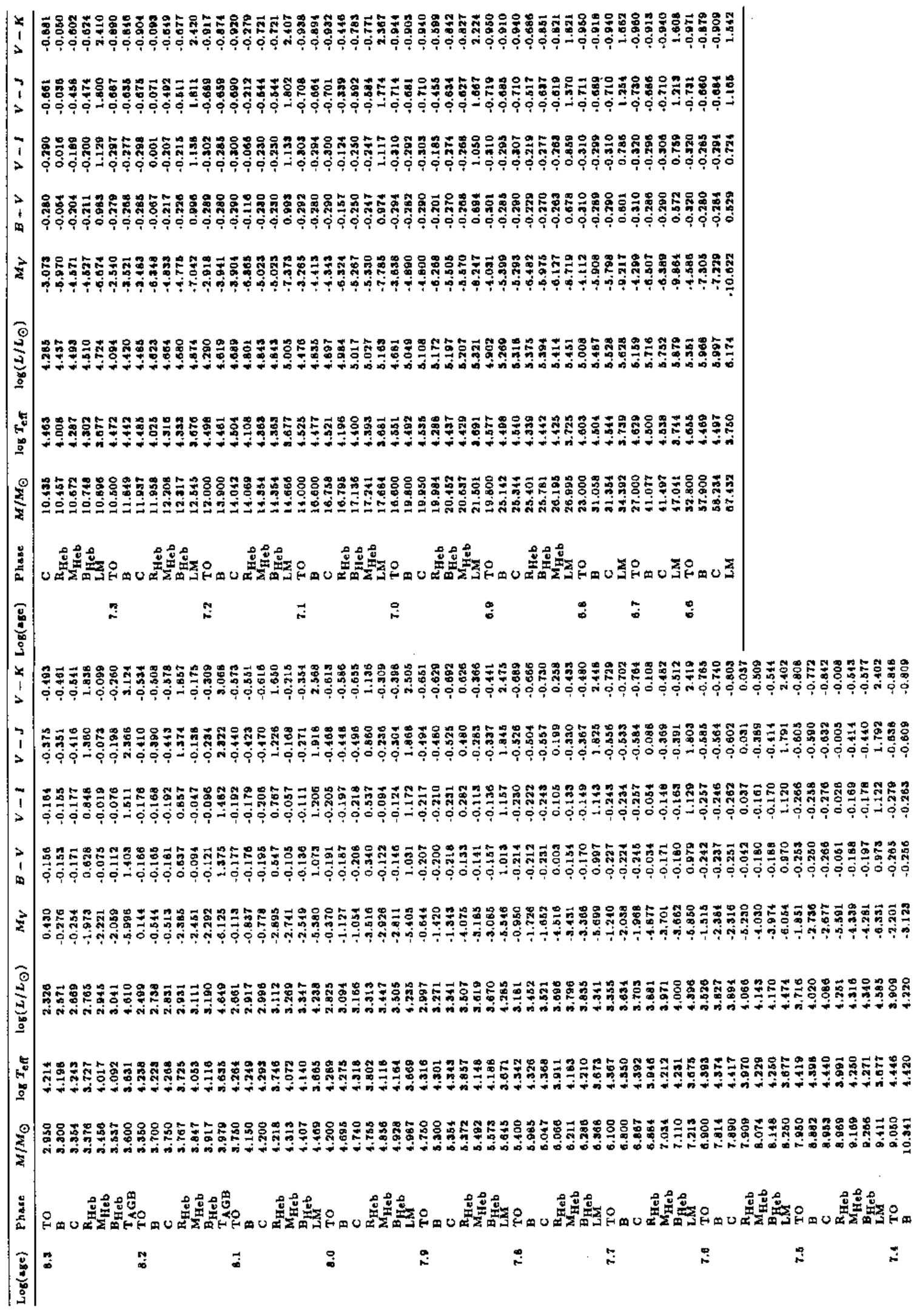

